\title{
sensors
}

ISSN 1424-8220

(C) 2006 by MDPI

http://www.mdpi.org/sensors

\section{Functional Testing and Characterisation of ISFETs on Wafer Level by Means of a Micro-droplet Cell}

Arshak Poghossian ${ }^{1,2}$, Kerstin Schumacher ${ }^{1,2}$, Joachim P. Kloock ${ }^{1,2}$, Christian Rosenkranz ${ }^{3}$, Joachim W. Schultze ${ }^{3}$, Mattea Müller-Veggian ${ }^{1}$ and Michael J. Schöning ${ }^{1,2, *}$

${ }^{1}$ Aachen University of Applied Sciences, Jülich Campus, Laboratory for Chemical Sensors and Biosensors, Ginsterweg 1, 52428 Jülich, Germany

${ }^{2}$ Institute of Thin Films and Interfaces (ISG-2), Research Centre Jülich GmbH, 52425 Jülich, Germany

${ }^{3}$ AGEF e.V.-Institute, Heinrich-Heine University, 40225 Düsseldorf, Germany

\# This paper dedicated in memory of Prof. Dr. Joachim W. Schultze.

* Author to whom correspondence should be addressed. Email: m.j.schoening@fz-juelich.de

Received: 28 July 2005 / Accepted: 4 January 2006 / Published: 7 April 2006

\begin{abstract}
A wafer-level functionality testing and characterisation system for ISFETs (ionsensitive field-effect transistor) is realised by means of integration of a specifically designed capillary electrochemical micro-droplet cell into a commercial wafer prober-station. The developed system allows the identification and selection of "good" ISFETs at the earliest stage and to avoid expensive bonding, encapsulation and packaging processes for nonfunctioning ISFETs and thus, to decrease costs, which are wasted for bad dies. The developed system is also feasible for wafer-level characterisation of ISFETs in terms of sensitivity, hysteresis and response time. Additionally, the system might be also utilised for wafer-level testing of further electrochemical sensors.
\end{abstract}

Keywords: ISFET, wafer-level testing, capillary micro-droplet cell. 


\section{Introduction}

Today, although well-established microelectronic mass-production techniques are used for the fabrication of microsensors and microsystem components, prices are still high. This is at least partly due to the fact that up to now the quality control and testing of microsensors and microsystem components is often carried out on chip-level, i.e. after dicing, bonding, encapsulation and mounting processes. This is an uneconomical approach, because the interconnection, encapsulation and packaging costs for microsystems can be very high, typically in the order of $55-85 \%$ of the total production costs $[1,2]$. These costs are further wasted for non-functioning chips (bad dies, see Figure 1). Therefore, testing at wafer level should be preferred in order to identify defects (shorted conducting paths, physically destroyed layers, conductor break, etc.) and to select those devices, which meet the predefined quality at the earliest stage (good dies), and thus, to avoid further expensive processes for bad dies (Figure 1). In the production of integrated circuits, the testing and quality control at wafer level is an established technology; different automatic wafer probers and testers are commercially available (see, e.g. [3]). Recently, wafer-level test solutions for several physical sensors, like pressure sensors, accelerometers and microphones, have been presented, too [4].

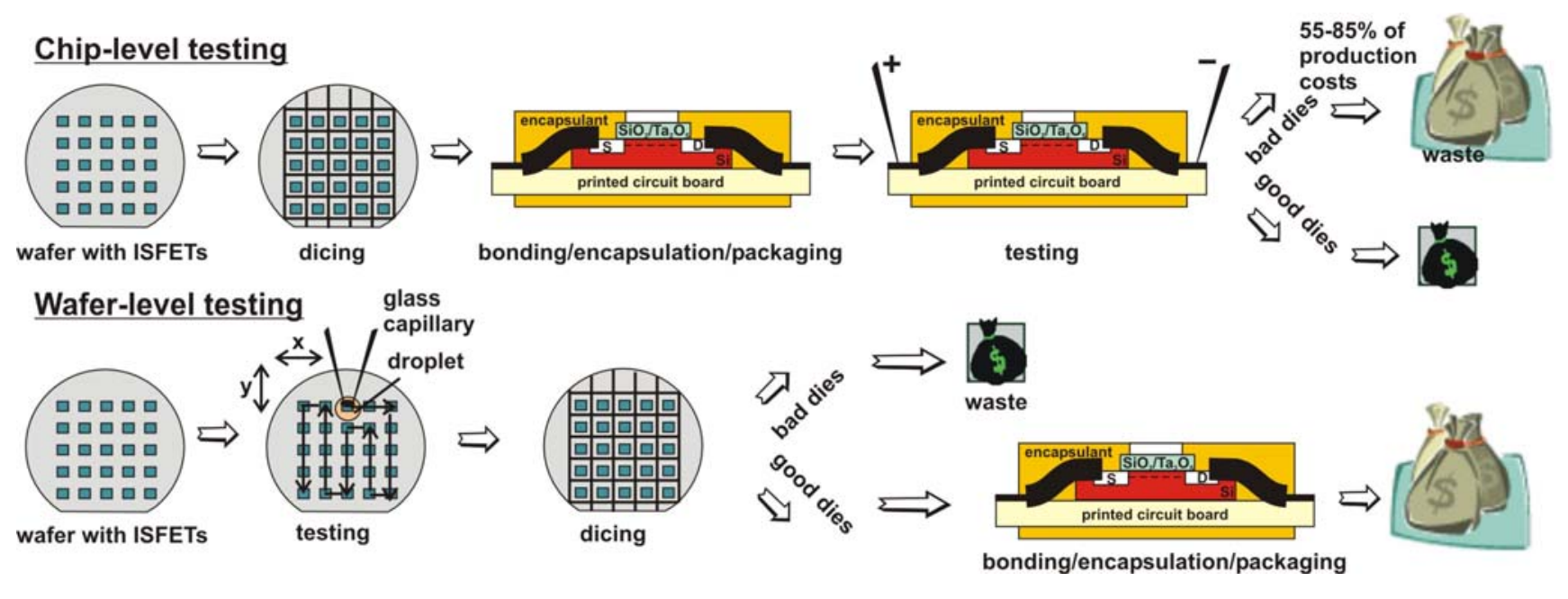

Figure 1. Wafer-level vs. chip-level testing.

On the other hand, there is an increasing demand for (bio-)chemical sensors and microanalysis systems such as $\mu$ TAS (micro total analysis system), lab-on-a-chip and electronic-tongue devices with a wide field of possible applications ranging from medicine, biotechnology and environmental monitoring over food and drug industries to defence and security purposes including antibioterrorism and biological warfare-agents field. Wafer-level testing of electrochemical microsensors, in particular ISFETs (ion-sensitive field-effect transistor) is much more difficult, because a non-electrical external stimulus such as liquid chemical substances should be applied to the gate of this device in order to verify its functionality. At the same time, the bonding pads or contact areas for electrical testing should be kept dry and isolated from the electrolyte solution. Minute electrolyte droplets could be dispensed (e.g., manually or using an ink-jet system) on the wafer in the gate region of the respective ISFET under test. Such a technique in combination with appropriate electrochemical techniques (e.g., cyclic voltammetry, electrochemical impedance spectroscopy and scanning electrochemical impedance 
spectroscopy) has been utilised for wafer-level quality control of microelectrodes [5]. In addition, a gel-based wafer-level testing procedure for microelectrodes has been suggested in [2]. However, due to the small gate sizes of ISFETs, special techniques are needed to reproducibly produce and control small droplets of a test sample. A capillary microcell [6,7], which is put directly onto the surface of interest (see Figure 2), could be a very useful technique to position tiny electrolyte droplets on the investigated surface and to provide spatially resolved electrochemical measurements on wafer level and in the micrometer scale.

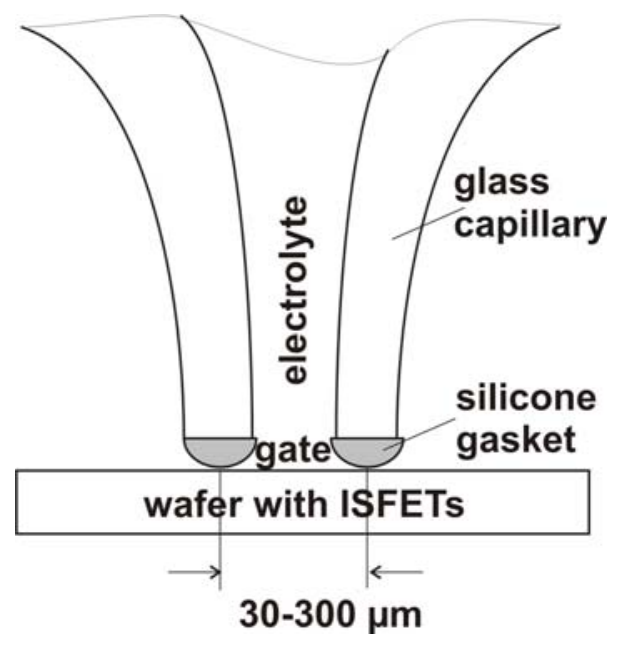

Figure 2. Capillary microcell for spatially resolved electrochemical measurements on wafer level. A silicone gasket at the tip of the capillary defines the testing area.

In this work, we report on the development and application of a specifically designed capillary electrochemical micro-droplet cell in combination with a commercial wafer prober-station to realise a wafer-level functionality testing and characterisation system for ISFET devices.

\section{Experimental}

The developed wafer-level testing system for ISFETs is shown in Figure 3. It combines the following basic parts: A commercial wafer prober-station (PM5 from Süss Microtec), a capillary micro-droplet cell, a semiconductor parameter analyzer (4155C from Agilent) or home made 4-channel ISFET-meter (not shown), a micromanipulator for the positioning of the microcell, and a microscope for optical control. An additional video microscope is used to control the droplet size, the contact between the surface and the filled glass capillary, and the position of the capillary onto the gate region. A constant mechanical contact pressure and thus, a reproducible wetted surface area has been achieved using a force sensor (KD45 from ME-Messsysteme GmbH, Germany), which monitors the force between the capillary tip and the wafer surface, digitizes the value and transfers it to the computer $[6,7]$. The computer can check whether the capillary is in contact with the wafer or not. 


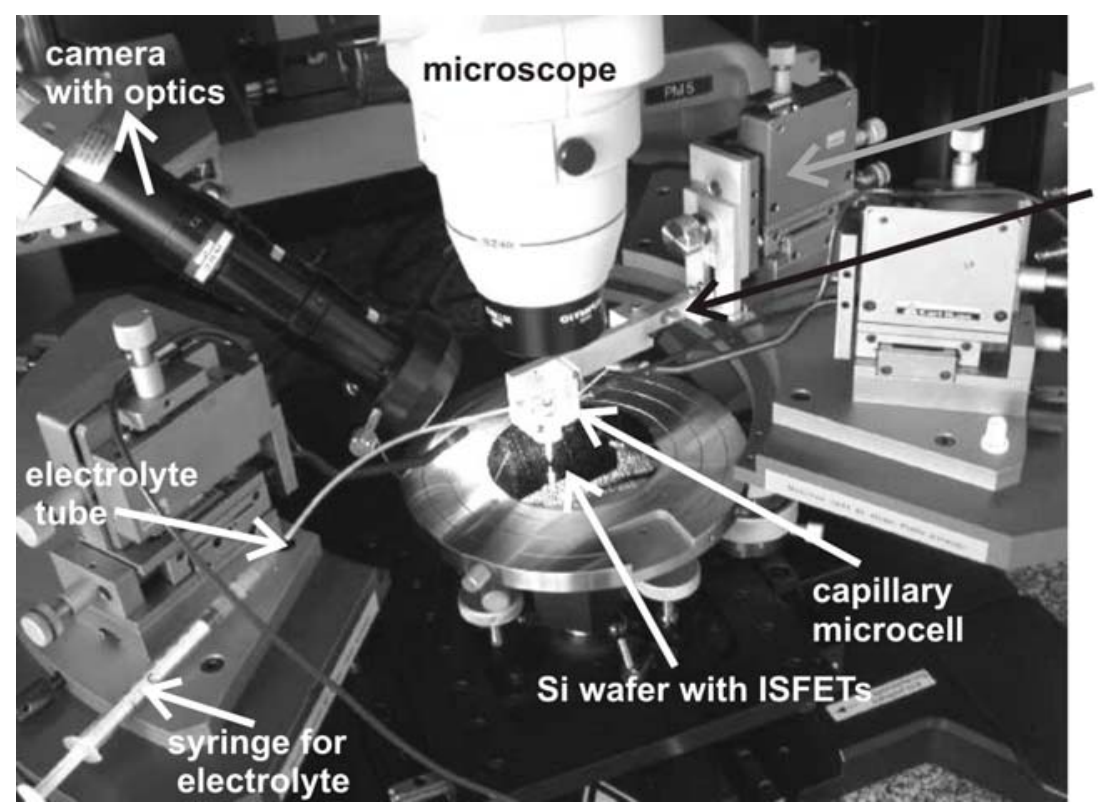

micro-manipulator

for the positioning

of the microcell

force sensor

provides a

reproducible

wetted surface

area

Figure 3. Photo of the ISFETs’ wafer-level testing system.

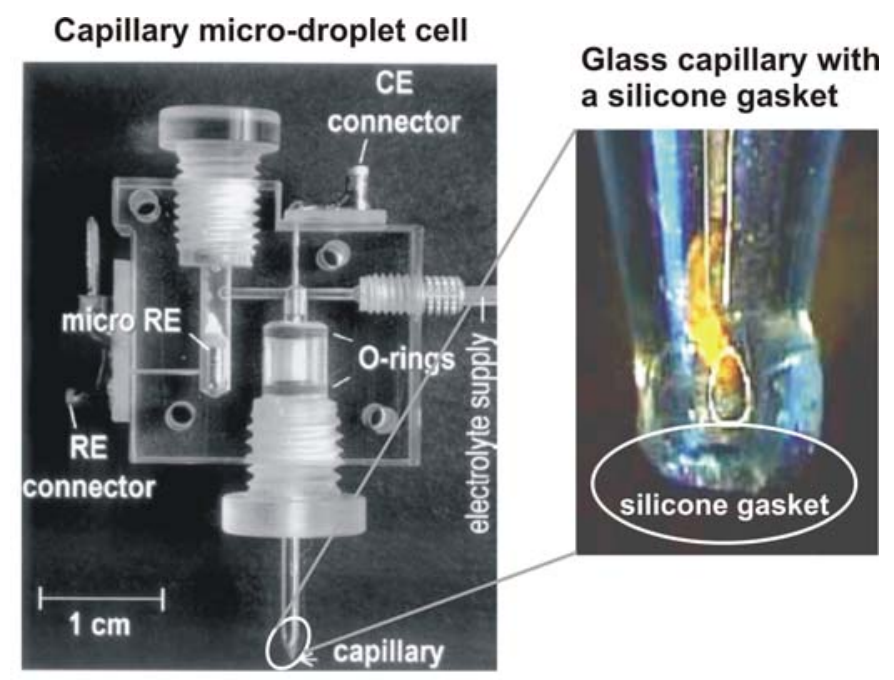

Figure 4. Capillary micro-droplet cell for testing of ISFETs on wafer level (RE: reference electrode, CE: counter electrode).

The designed capillary micro-droplet plexiglass-cell is based on a conventional three-electrode arrangement (Figure 4). The reference ( $\mathrm{Au}$ wire or $\mathrm{Hg} / \mathrm{HgCl}_{2}$ ) and counter ( $\mathrm{Au}$ wire) electrodes are connected by the glass capillary filled with the electrolyte. A silicone gasket has been formed on the flat glass-capillary tip, which has been prepared by previous laser pulling and mechanical polishing. The hydrophobic surface of the silicone gasket prevents leakage of the electrolyte. Prior to measurements, a droplet of an electrolyte solution is applied on the die under test, exclusively covering the gate region of the respective ISFET. The contact area of the gate with the electrolyte is defined by the diameter of the silicone gasket. Because of the adjustable geometry of the tip size of the capillary, the rest of the ISFET has no contact to the electrolyte. 


\section{Results and discussion}

The system has been tested using wafers combining n-channel ISFETs with different geometrical sizes and gate layouts (linear-, meander- and U-shaped). The layouts of the ISFETs used are presented in Figure 5. The double-layer gate insulator consists of $\mathrm{SiO}_{2}(80 \mathrm{~nm}) / \mathrm{Ta}_{2} \mathrm{O}_{5}(80 \mathrm{~nm})$ (layout (a) and (b)) and $\mathrm{SiO}_{2}(30 \mathrm{~nm}) / \mathrm{Si}_{3} \mathrm{~N}_{4}\left(70 \mathrm{~nm}\right.$ ) (layout (c)) films. The $\mathrm{pH}$-sensitive $\mathrm{Ta}_{2} \mathrm{O}_{5}$ and $\mathrm{Si}_{3} \mathrm{~N}_{4}$ layers have been prepared by thermal oxidation of sputtered Ta (40 nm thick) in a dry oxygen atmosphere for about $2 \mathrm{~h}$ and PECVD (plasma-enhanced chemical vapour deposition) method, respectively. The basic characteristics of these ISFETs (but encapsulated) have been reported previously (see e.g., [8-10]).

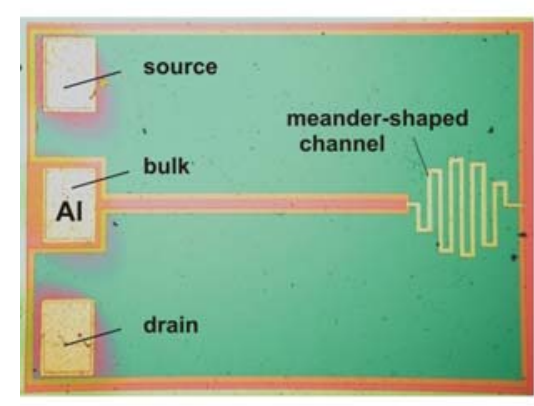

a)

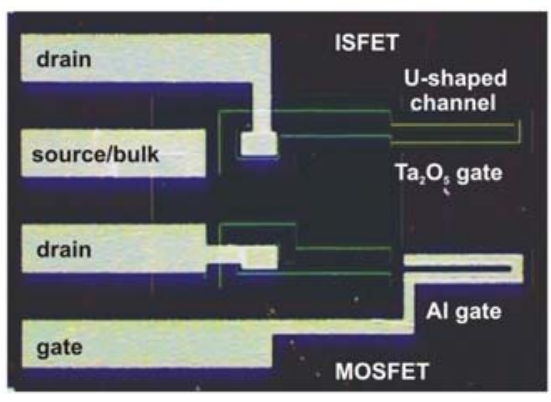

b)

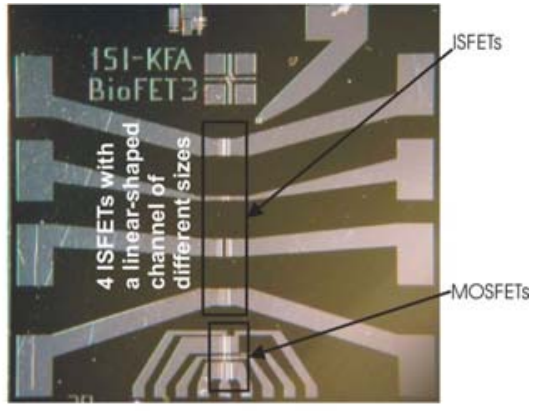

c)

Figure 5. Layouts of different ISFET chips consisting of a single ISFET with a meander-shaped channel (a), an ISFET/MOSFET (metal-oxide-semiconductor FET) pair with an U-shaped channel (b) and four ISFETs and one MOSFET with a linear-shaped channel (c).

To test the functionality of the ISFETs, a buffer droplet of $\mathrm{pH} 7$ was applied to the gate area of the selected ISFET on the wafer by means of the micro-droplet cell, and a set of output and transfer curves has been recorded using a semiconductor parameter analyzer or a home-made ISFET-meter. Dependent on the ISFET layout and the size of the gate area (see Figure 5), capillaries with different diameters have been used. As an example, Figure 6 exemplarily shows a typical set of output (a) and transfer (b) curves of a U-shaped $\mathrm{Ta}_{2} \mathrm{O}_{5}$-gate ISFET (layout (b) in Figure 5). They are comparable to those that have been measured on chip-level with fully encapsulated ISFETs, i.e. after the complete fabrication process.

In addition to functionality tests, the developed system allows also a wafer-level characterisation of ISFETs in terms of $\mathrm{pH}$ sensitivity or hysteresis by applying buffer droplets with different $\mathrm{pH}$ values to the gate surface. Prelimanary experiments have verified the possibility to perform such measurements with the developed micro-droplet cell. As an example, Figure 7 represents a constant-charge-mode dynamic response for a meander-shaped $\mathrm{Ta}_{2} \mathrm{O}_{5}$-gate ISFET (layout (a) in Figure 5) in buffer solutions of different $\mathrm{pH}$ values from $\mathrm{pH} 3$ to $\mathrm{pH} 9$ measured on wafer level by means of the developed experimental set-up with the micro-droplet cell. The $\mathrm{pH}$ sensitivity evaluated from Figure 7 was about $50 \mathrm{mV} / \mathrm{pH}$ (in comparison to $55-58 \mathrm{mV} / \mathrm{pH}$ for the encapsulated $\mathrm{Ta}_{2} \mathrm{O}_{5}$-ISFET [8]). At the same time, a relatively large hysteresis and a longer response time have been observed that can be attributed to rests of the previous buffer solution due to difficulties of careful rinsing of the capillary and the ISFET-gate surface as well as due to the possible presence of micro-bubbles in the gate region. These disadvantages could be avoided by using a flow-through capillary micro-droplet cell, which has been 
recently proposed in [7]. Here, a continuous electrolyte flow might allow an easy and careful rinsing of the micro-droplet cell and the gate surface as well as a faster change of different $\mathrm{pH}$ buffer solutions.
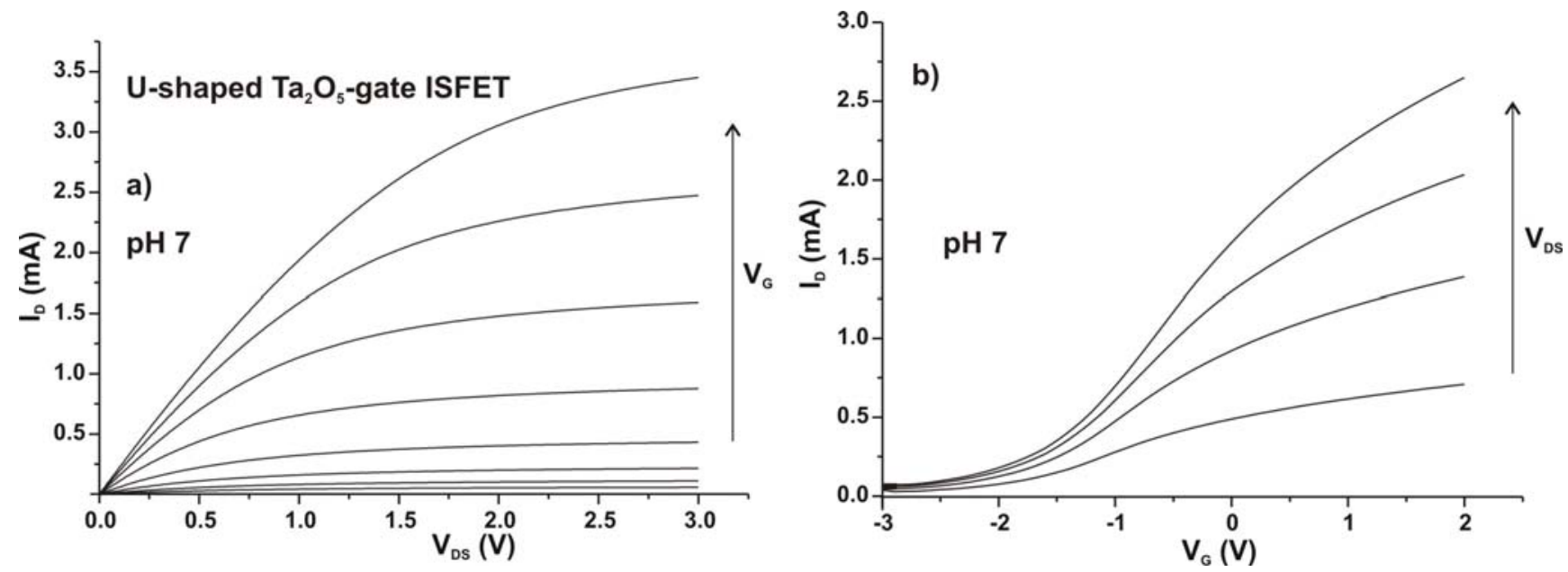

Figure 6. Output (a) and transfer (b) characteristics of an ISFET (layout (b) in Figure 5) measured on wafer level by means of the developed experimental set-up with the micro-droplet cell.

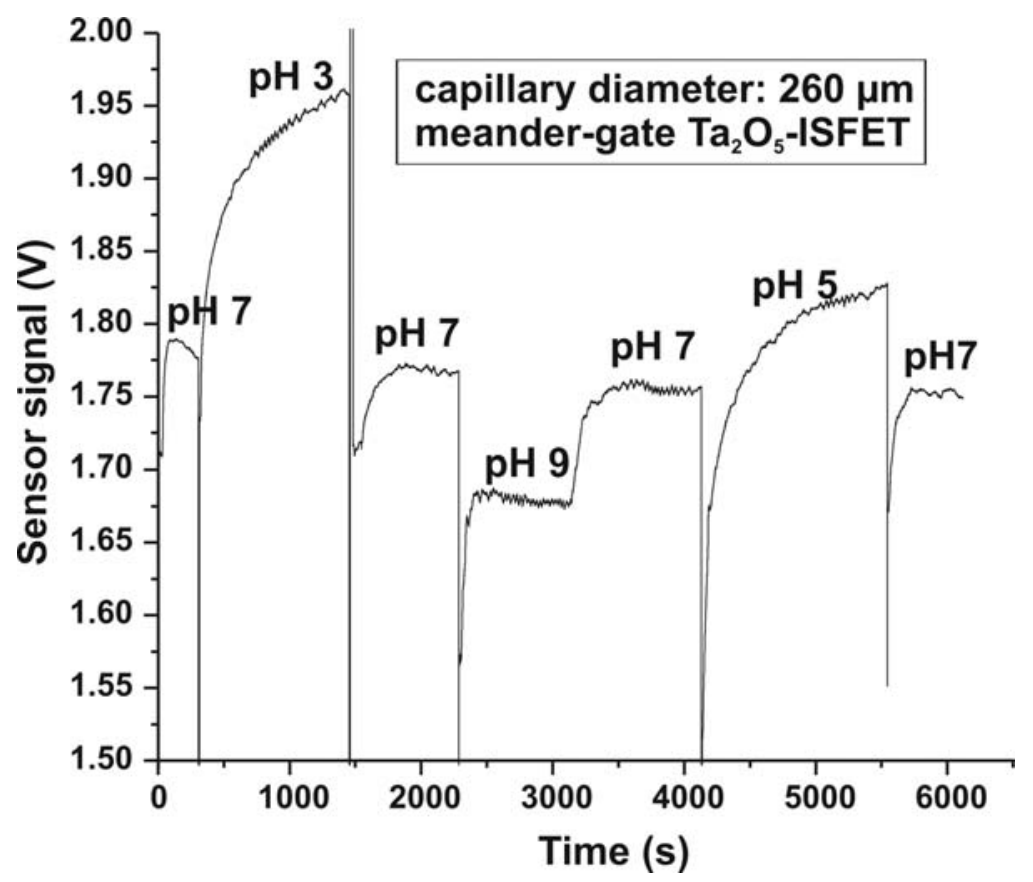

Figure 7. Constant-charge-mode dynamic response of a meander-shaped $\mathrm{Ta}_{2} \mathrm{O}_{5}$-ISFET (layout (a) in Figure 5) in buffer solutions of different $\mathrm{pH}$ values from $\mathrm{pH} 3$ to $\mathrm{pH} 9$ measured at wafer level by means of the developed experimental set-up with the micro-droplet cell.

An ISFET is one of the basic structural elements of a new generation of chemical sensors and biosensors. At present, ISFET-based sensors and sensor arrays have been successfully realised for the detection of $\mathrm{pH}$, concentration of different ions and analytes as well as for the measurement of even action potentials of living cells (see, e.g., review [11]). Recently, several attempts have been also made to apply field-effect transistors for the detection of macromolecular interactions such as antibodyantigen binding, DNA (deoxyribonucleic acid) hybridisation, etc. [12-14]. In addition, the possibility 
of application of ISFETs for the detection of physical quantities, like temperature, flow rate, flow direction, diffusion coefficient and liquid level, has been demonstrated, too [8,15]. Moreover, ISFETs are now commercial reality. pH-sensitive ISFETs are available from more than 20 companies such as Orion (USA), Beckman Coulter (USA), Sentron Europe (The Netherlands), Honeywell (USA), Endress+Hausser (Germany), Mettler Toledo (Switzerland), Horiba (Japan) and other leading producers of electrochemical sensors. The technological process of fabrication of those ISFETs has been achieved to such a level, that ISFETs exhibit performances, which are comparable with those of glass pH-electrodes [16]. Furthermore, sensor-check systems allowing to inspect automatically the sensor operation during use, are now commercially available for the ISFET pH-sensor from Endress+Hauser [16]. However, to our knowledge, there is no commonly accepted procedure and commercially available equipment for a wafer-level functionality testing and characterisation of ISFETs so far. In this context, the developed system for functionality testing and characterisation of ISFETs on wafer level could be a very attractive tool for both ISFET producers and ISFET users.

\section{Conclusions}

A wafer-level functionality testing system for ISFETs has been realised by means of integration of a specifically designed capillary electrochemical micro-droplet cell into a commercial wafer proberstation. The obtained experimental results demonstrate also the feasibility of the developed system for the wafer-level characterisation of ISFETs in terms of sensitivity and hysteresis. The developed system allows to identify and select "good" ISFETs at the earliest stage of the fabrication process and to avoid expensive bonding, encapsulation and packaging processes for non-functioning ISFETs and thus, to decrease costs, which are wasted for bad dies. In addition, a single non-encapsulated ISFET can be tested and characterised. Furthermore, the system might be also utilised for wafer-level testing of further electrochemical sensors. In the future, we plan to automatically scan the microcell over the whole wafer surface (as it is shown in Figure 1) for a fast testing of all sensor structures.

\section{Acknowledgements}

Parts of this work were supported by the Ministerium für Innovation, Wissenschaft, Forschung und Technologie des Landes Nordrhein-Westfalen.

\section{References}

1. Mehalso, R. Packaging of microsystems. Proceedings of the Micromechanics Europe Workshop, Cork, Ireland, September 16-18, 2001, pp. 98-110.

2. Kindlundh, M.; Norlin, P. A gel-based wafer-level testing procedure for microelectrodes. Sens. Actuators B, 2005, 107, 557.

3. http://www.cascademicrotech.com.

4. Bay, J.; Branebjerg, J. Functional testing of micromachined mechanical sensors on wafer level. Proceedings of the Eurosensors-14, Copenhagen, Denmark, August 27-30, 2000, pp. 317-318. 
5. Köster, O.; Schuhmann, W.; Vogt, H.; Mokwa, W. Quality control of ultra-microelectrode arrays using cyclic voltammetry, electrochemical impedance spectroscopy and scanning electrochemical microscopy. Sens. Actuators B, 2001, 76, 573.

6. Lohrengel, M.M.; Moehring, A.; Pilaski, M. Capillary-based droplet cells: Limits and new aspects. Electrochim. Acta, 2001, 47, 137.

7. Lohrengel, M.M.; Rosenkranz, C.; Klüppel, I.; Moehring, A.; Bettermann, H.; Van den Bossche, B.; Deconinck, J. A new microcell or microreactor for material surface investigations at large current densities. Electrochim. Acta, 2004, 49, 2863.

8. Poghossian, A.; Schöning, M.J. Detecting both physical and (bio-)chemical parameters by means of ISFET devices. Electroanalysis, 2004, 16, 1863.

9. Poghossian, A.; Lüth, H.; Schultze, J.W.; Schöning, M.J. (Bio-)chemical and physical microsensor arrays using an identical transducer principle. Electrochim. Acta, 2001, 47, 243.

10. Schroth, P.; Schöning, M.J.; Kordos, P.; Lüth, H.; Schütz, S.; Weißbecker, B.; Hummel, H.E. Insect-based BioFETs with improved signal characteristics. Biosens. Bioelectron., 1999, 14, 303.

11. Schöning, M.J.; Poghossian, A. Recent advances in biologically sensitive field-effect transistors (BioFETs). Analyst, 2002, 127, 1137.

12. Uslu, F.; Ingebrandt, S.; Mayer, D.; Böcker-Meffert, S.; Odenthal, M.; Offenhäusser, A. Labelfree fully electronic nucleic acid detection system based on a field-effect transistor device. Biosens. Bioelectron., 2004, 19, 1723.

13. Pouthas, F.; Gentil, C.; Cote, D.; Bockelmann, U. DNA detection on transistor arrays following mutation-specific enzymatic amplification. Appl. Phys. Lett., 2004, 84, 1594.

14. Sakata, T.; Kamahori, M.; Miyahara, Y. DNA analysis chip based on field-effect transistors. Jpn. J. Appl. Phys., 2005, 44, 2854.

15. Poghossian, A.; Berndsen, L.; Schöning, M.J. Chemical sensor as physical sensor: ISFET-based flow-velocity, flow-direction and diffusion-coefficient sensor. Sens. Actuators B, 2003, 95, 384.

16. Oelßner, W.; Zosel, J.; Guth, U.; Pechstein, T.; Babel, W.; Connery, J.G.; Demuth, C.; Grote Gansey, M.; Verburg, J.B. Encapsulation of ISFET sensor-chips. Sens. Actuators B, 2005, 105, 104.

(C) 2006 by MDPI (http://www.mdpi.org). Reproduction is permitted for non-commercial purposes. 